\title{
Effects of insulin-like growth factor binding protein 3 on cell growth and tumorigenesis in oral squamous cell carcinoma
}

\author{
Hong-Yuan $\mathrm{Xu}^{1,2,3}$, Dong-Wang Zhu ${ }^{2,4}$, Lai-Ping Zhong ${ }^{2,3,4}$, Zhi-Yuan Zhang ${ }^{2,3,4}$, Cheng-Zhe Yang ${ }^{5}$, \\ Xiao Yang ${ }^{1,2,3}$, Peng Zhang ${ }^{2,3,6}$
}

${ }^{1}$ Center of Craniofacial Orthodontics, Department of Oral and Cranio-Maxillofacial Science, Ninth People's Hospital, Shanghai Jiao Tong University School of Medicine, Shanghai 200011, China; ${ }^{2}$ Shanghai Key Laboratory of Stomatology \& Shanghai Research Institute of Stomatology, National Clinical Research Center of Stomatology, Shanghai 200011, China; ${ }^{3}$ National Clinical Research Center for Oral Diseases, ${ }^{4}$ Department of Oral \& Maxillofacial-Head \& Neck Oncology, Ninth People's Hospital, Shanghai Jiao Tong University School of Medicine, Shanghai 200011, China; ${ }^{5}$ Department of Oral \& Maxillofacial Surgery, Qilu Hospital and Institute of Stomatology, Shandong University, Jinan 250012, China; ${ }^{6}$ The 2nd Dental Center, Ninth People's Hospital, Shanghai Jiao Tong University School of Medicine, Shanghai 200011, China

Contributions: (I) Conception and design: X Yang, P Zhang; (II) Administrative support: L Zhong, Z Zhang; (III) Provision of study materials or patients: C Yang; (IV) Collection and assembly of data: D Zhu; (V) Data analysis and interpretation: H Xu; (VI) Manuscript writing: All authors; (VII) Final approval of manuscript: All authors.

Correspondence to: Xiao Yang. Center of Craniofacial Orthodontics, Department of Oral and Cranio-Maxillofacial Science, Ninth People's Hospital, Shanghai Jiao Tong University School of Medicine, No. 639 Zhizaoju Road, Shanghai 200011, China. Email: xiaozhu09901@sina.com; Peng Zhang. The 2nd Dental Center, Ninth People's Hospital, Shanghai Jiao Tong University School of Medicine, No. 639 Zhizaoju Road, Shanghai 200011, China. Email: zp_kitty@163.com.

Background: This study was performed to investigate the effect of insulin-like growth factor binding protein 3 (IGFBP3) on the biological behavior of tumor cells and tumorigenesis of oral squamous cell carcinoma (OSCC).

Methods: OSCC HB96, CAL27, and Tca8113 cells were transfected with the following plasmids: siRNAIGFBP3, pcDNA-0-IGFBP3, or siRNA-NC (negative control). The effect of aberrant IGFBP3 on cell viability, apoptosis, and colony formation was assessed. Quantitative real-time PCR (qRT-PCR) and western blot analysis were used to measure IGFBP3 mRNA and protein levels, respectively. HB96 and CAL27 cells were transfected with IGFBP3-expressing lentiviral plasmids and then transplanted into nude mice to monitor xenograft tumor formation.

Results: An optimal transfection efficiency was obtained with 50 pmol siRNA-IGFBP3. Transient silencing of IGFBP3 significantly reduced cell viability, and increased apoptosis in comparison with the non-targeting negative control (NC). Overexpressing IGFBP3 promoted cell viability. Additionally, in comparison with the NC group, both cell growth and colony formation were reduced, while apoptosis was elevated in stably transfected cells. Moreover, silencing IGFBP3 inhibited cell viability and tumor formation in nude mice after 3 weeks, and colony formation, diminished tumorigenesis in nude mice, but promoted cell apoptosis in OSCC cells.

Conclusions: Collectively, our study revealed a protumorigenic role for IGFBP3 in OSCC cancer cells, and demonstrated a potential mechanism for the dysregulation of IGFBP3 in cell growth. Therefore, IGFBP3 may be a potential therapeutic target for the treatment of OSCC.

Keywords: Insulin-like growth factor binding protein 3 (IGFBP3); oral squamous cell carcinoma (OSCC); tumorigenesis; small interfering RNA

Submitted Jul 23, 2018. Accepted for publication Jul 30, 2019.

doi: $10.21037 /$ tcr.2019.08.13

View this article at: http://dx.doi.org/10.21037/tcr.2019.08.13 


\section{Introduction}

Oral cancer is one of the top ten common malignancies observed in the human body, which results in dysfunction in eating, speaking, and breathing. Oral squamous cell carcinoma (OSCC), accounts for $90 \%$ of oral cancer cases and is the most prevalent tumor in the head and neck area. OSCC is characterized by poor patient prognosis, with a 5 -year survival rate of $50-60 \%(1,2)$. Interestingly, $50 \%$ of OSCC patients exhibit cervical lymph node metastasis, which has been suggested to be a prognostic indicator of poor clinical outcomes $(3,4)$. In spite of the numerous advances in both diagnosis and treatment, including radical surgery, radiotherapy, and neoadjuvant chemotherapy, the past decade has seen little significant improvement in the prognosis or survival of patients with advanced or recurrent OSCC (5). Thus, a better understanding of the OSCC pathogenesis and the development of predictive and prognostic biomarkers are the immediate needs of OSCC patients.

Insulin-like growth factor binding protein 3 (IGFBP3) is a principal member of the IGFBP family that can bind with circulating insulin-like growth factor (IGF), which regulates the mitogenic activity (6). There is overwhelming evidence corroborating the key role of IGFBP3 in the pathogenesis and progression of cancers. IGFBP 3 expression is upregulated in most tumors, including pancreatic ductal adenocarcinoma, gastrointestinal stromal tumor, lung, gastric, and breast cancers (7-10). However, reduced IGFBP3 expression has been reported in hepatocellular carcinoma (HCC) (11). Zhong et al. demonstrated the transcriptomic and proteomic overexpression of IGFBP3 in OSCC cell lines and human tumor tissues (12). Functionally, IGFBP3 has been shown to act as an antioxidant by inhibiting reactive oxygen species. It has also been shown to promote epithelial-to-mesenchymal transition and motility $(13,14)$. IGFBP3 has prognostic value in various cancers including breast, colorectal, and prostate cancers. Yen et al. also demonstrated that IGFBP3 elevated the migration and lymph node metastasis of OSCC cells in an IGF-independent pattern (15). Therefore, IGFBP3 is likely to be a promoter of tumorigenesis. Nevertheless, the functional role of IGFBP3 in OSCC still remains unclear.

To our knowledge, there are only a few reports that have detailed IGFBP3 function in OSCC in vitro and in vivo. In the present study, we used small interfering RNA (siRNA) and gene cloning technology to silence and overexpress IGFBP3 by transient and stable transfection of OSCC cell lines. The functional role of IGFBP3 in cell viability, apoptosis, colony formation in vitro, and tumorigenesis in vivo was evaluated. We established the relationship between IGFBP 3 and cell growth and tumorigenesis of OSCC. This is expected to further define OSCC pathogenesis and spark the development of better therapeutic strategies for the clinical management of the disease.

\section{Methods}

\section{Cell culture}

Ninth People's Hospital, Shanghai Jiao Tong University School of Medicine Human OSCC cell lines, HB96 and CAL27 (American Type Culture Collection, Manassas, USA), were grown in Dulbecco's Modified Eagle's Medium (DMEM, Gibco, USA), supplemented with 10\% fetal bovine serum (FBS; Gibco, Grand Island, USA), $100 \mathrm{U} / \mathrm{mL}$ penicillin and $100 \mu \mathrm{g} / \mathrm{mL}$ streptomycin. Human OSCC Tca8113 cells were cultivated in RPMI1640 medium (Gibco, Grand Island, USA), supplemented with $10 \% \mathrm{FBS}, 100 \mathrm{U} / \mathrm{mL}$ penicillin and $100 \mu \mathrm{g} / \mathrm{mL}$ streptomycin. All three OSCC cell lines were kept at $37{ }^{\circ} \mathrm{C}$ in a humidified incubator with $5 \% \mathrm{CO}_{2}$. Cells were grown to $70-80 \%$ confluence and then used for experiments.

\section{IGFBP3 stable transfection}

The IGFBP3 overexpression plasmid (pcDNA3.0-IGFBP3) and its control vector plasmid were preserved in our laboratories. Short hair IGFBP3 shRNA synthesized by Sangon Biotech (Shanghai, China) was inserted into the pSUPER via enzyme digestion, and the IGFBP3 shRNA plasmid was constructed through transfection, selection, and plasmid extraction. The target sequence for IGFBP3 shRNA is as follows: 5'-GCACAGATACCCAGAACTT-3'. HB96, CAL27, and Tca8113 cells were transfected with corresponding plasmids to overexpression or knockdown IGFBP3 using Lipofectamine 2000 reagent according to the manufacturer's instructions, and incubated at $37^{\circ} \mathrm{C}$ for $48 \mathrm{~h}(16-18)$.

\section{Western blot}

Total protein was isolated from HB96, CAL27, and Tca8113 cells using a lysis buffer (Applygen Technologies Inc., Beijing, China). Protein concentration was then determined 
using a BCA Protein Assay Kit (PIERCE, USA). Samples were then transferred to polyvinylidene fluoride (PVDF) membrane (Millipore, Boston, USA). Subsequently, membranes were incubated with the primary antibody (antiIGFBP3 polyclonal antibody, Abcam, Cambridge, UK; 1:1,000) overnight at $4{ }^{\circ} \mathrm{C}$. After washing with phosphate buffered saline (PBS) three times, membranes were incubated with a horseradish peroxidase-tagged secondary antibody (Fermentas, Vilnius, Lithuania; 1:1,000) for $1 \mathrm{~h}$ at room temperature. $\beta$-Actin was used as the loading control. The bands were visualized with an Odyssey laser scanning system.

\section{Cell viability assay}

OSCC cell growth was measured by cell counting kit-8 (CCK-8; Dojindo, Kumamoto, Japan). Briefly, HB96, CAL27, and Tca8113 cells $\left(2 \times 10^{3} /\right.$ well $)$ were seeded in 96-well microplates. Afterwards, $10 \mu \mathrm{L}$ CCK-8 solution was added and cells were incubated for $1 \mathrm{~h}$ at $37{ }^{\circ} \mathrm{C}$ in a humidified $5 \% \mathrm{CO}_{2}$ incubator. The absorbance was assessed on an automatic quantitative microplate reader (Anthos Labtec Instruments GmbH, Wals, Austria) at $450 \mathrm{~nm}$ for 7 consecutive days.

\section{Apoptosis assay}

Apoptosis of OSCC cells in the NC and si-IGFBP3 groups were quantified using Annexin V/7-AAD staining. Cellular supernatants from OSCC cell cultures were collected in flow tubes and fixed with $1 \%$ pancreatin. Fixed cells were then resuspended in $0.5 \mathrm{~mL}$ binding buffer and counted. Annexin $\mathrm{V}$ and 7 -AAD $(2.5 \mu \mathrm{L}$ each) were then added to $100 \mu \mathrm{L}$ aliquots of the fixed cells and incubated for $15 \mathrm{~min}$. The cells were then analyzed by flow cytometry.

\section{Colony formation assay}

OSCC cells in the NC and si-IGFBP3 groups were trypsinized and then cultured at a density of 1,000 cells $/ 10 \mathrm{~cm}$ in RPIM-1640 medium supplemented with a working concentration of $600 \mathrm{mg} / \mathrm{mL} \mathrm{G418} \mathrm{for} 3$ weeks. Cells were fixed using cold methanol and glacial acetic acid (3:1) for $30 \mathrm{~min}$ and stained with Gimsa (Sigma, USA) for $20 \mathrm{~min}$. The surviving colonies (number of cells $\geq 50$ ) were manually counted and photographed.

\section{Lentivirus-mediated gene transfer}

The shuttle vector SI505A-1 was ligated with IGFBP3siRNA-516 to construct the plasmid pGreenPuroshIGFBP3. The packaging procedure was performed based on SBI's user manual. SBI lentivirus system contained four plasmids pPACKH1-REV, pPACKH1-GAG, PVSV-G and pGreenPuro-shIGFBP3 along with green fluorescent protein (GFP; Open Biosystems, Huntsville, USA). The four plasmids were cotransfected into HB96 and CAL27 cells at $50-70 \%$ confluence.

\section{Xenograft transplantation experiments}

All the animal experimental protocols were approved by the ethics committee of Shanghai Ninth People's Hospital, Shanghai Jiao Tong University School of Medicine [HKDL(2018)410]. In brief, $5 \times 10^{6} / \mathrm{mL}$ of CAL27 cells were implanted subcutaneously in quadruplicate fashion into BALB/cASlac-nu mice (male, 3-4 weeks old; Laboratory Animal Center of Chinese Academy of Science, Shanghai, China). The tumor volume was measured. Mice were euthanized 5 weeks post tumor implantation, and tumors were excised for gross observation and measurement of tumor weight.

\section{Statistical analysis}

Data are expressed as mean \pm standard deviation (SD). Statistical differences were evaluated using SPSS 19.0 (SPSS, IL, USA). Statistical analysis was performed using one-way analysis of variance. We considered a $\mathrm{P}$ value of $<0.05$ as statistically significant.

\section{Results}

\section{Transfection efficiency of IGFBP3 knockdown in HB96, CAL27, and Tca8113 cells}

Preliminary transfection experiments were performed in Tca8113 cells using 20, 50, and 100 pmol IGFBP3-targeting siRNA in order to determine the siRNA concentration necessary for efficient knockdown of IGFBP3 (Figure 1A). Twenty-four hours after transfection, nearly $80 \%$ of Tca8113 cells expressed green fluorescence protein (GFP) in response to $50 \mathrm{pmol}$ siRNA. Additionally, qRT-PCR results showed that si-IGFBP3 significantly reduced IGFBP3 

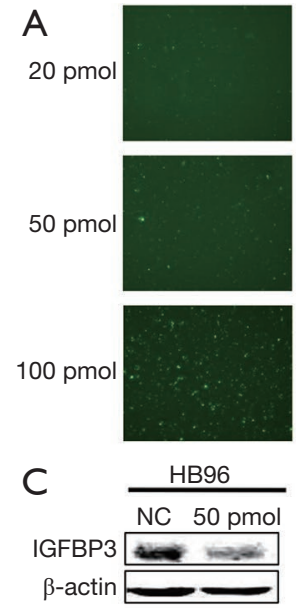

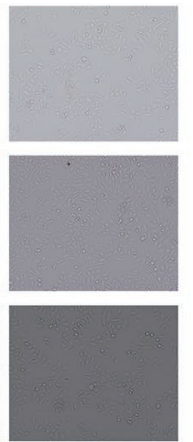

B

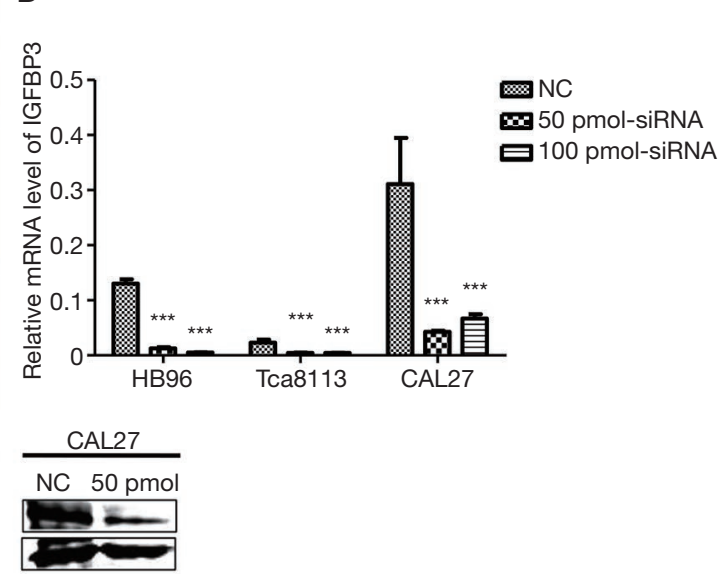

Figure 1 Transfection efficiency of siRNA-IGFBP3 in HB96, CAL27, and Tca8113 cells. (A) Transient knockdown of IGFBP3 was performed with 20, 50, and 100 pmol siRNA in Tca8113 cells; (B) the mRNA level of IGFBP3 was decreased by 50 and 100 pmol siIGFBP3; (C) the protein level of IGFBP3 was decreased by 50 pmol si-IGFBP3. siRNA, small interfering RNA; IGFBP3, insulin-like growth factor binding protein $3 .{ }^{* * *}, \mathrm{P}<0.001$.

mRNA levels compared with siRNA-NC (Figure 1B). The interference effect of 50 and 100 pmol siRNA was similar. Due to the cytotoxicity of siRNA, $50 \mathrm{pmol}$ siRNA was selected as the optimal dose for IGFBP3 silencing. The data shown in Figure $1 C$ demonstrates that IGFBP3 protein was potently reduced by si-IGFBP3 in comparison to siRNANC. Therefore, 50 pmol si-IGFBP3 was the concentration chosen to be used in our subsequent experiments, for efficient knockdown of IGFBP3 in HB96, CAL27, and Tca8113 cells.

\section{Effect of IGFBP3 knockdown on cell viability and apoptosis in HB96, CAL27, and Tca8113 cells}

The effect of IGFBP3 knockdown on cell viability and apoptosis in HB96, CAL27, and Tca8113 cells is shown in Figure 2. We found that cell viability with IGFBP3 knockdown was remarkably lower than that of the $\mathrm{NC}$ group in the HB96, CAL27, and Tca8113 cells (Figure 2A). In the HB96 cell line, there was a significant increase in the number of apoptotic cells after transfection of the IGFBP3targeting siRNA (Figure 2B). These findings indicate that silencing IGFBP3 inhibited cell growth in OSCC cells.

\section{Effect of stable IGFBP3 knockdown on Tca8113 cell functions}

Since transiently silencing IGFBP3 can inhibit cell growth, we further detected the effect of stable transfection by using a short hairpin lentiviral plasmid pSUPER-IGFBP3shRNA-516. After transfecting pSUPER-IGFBP3shRNA-516 into Tca 8113 cells, western blot analysis showed that the expression of IGFBP3 protein was noticeably decreased in cells expressing the IGFBP3-interfering plasmid in comparison to cells expressing the non-targeting plasmid (Figure 3A). Subsequently, we assessed cell viability, apoptosis, and colony formation in stable Tca8113 cells. Figure 3B,C,D shows that cell growth was slower, cell apoptosis was elevated, and colony formation was reduced in stably transfected cells in comparison with control cells. These data suggest that silencing IGFBP3 suppressed tumor cell growth of OSCC cells.

\section{Effect of lentivirus-mediated overexpression of IGFBP3 on tumorigenesis}

We transfected IGFBP3 lentiviral plasmids into HB96 and CAL27 cells to determine the effect of silencing IGFBP3 on OSCC tumorigenesis. We found that the expression of IGFBP3 protein was significantly decreased in comparison to the control (Figure 4A). In mice, CAL27 cells with silencing of IGFBP3 were implanted subcutaneously, and tumor size and weight were measured (Figure 4B). We found that the tumor size was smaller than the control after 3 weeks. Thus, silencing IGFBP3 inhibited tumor growth. 

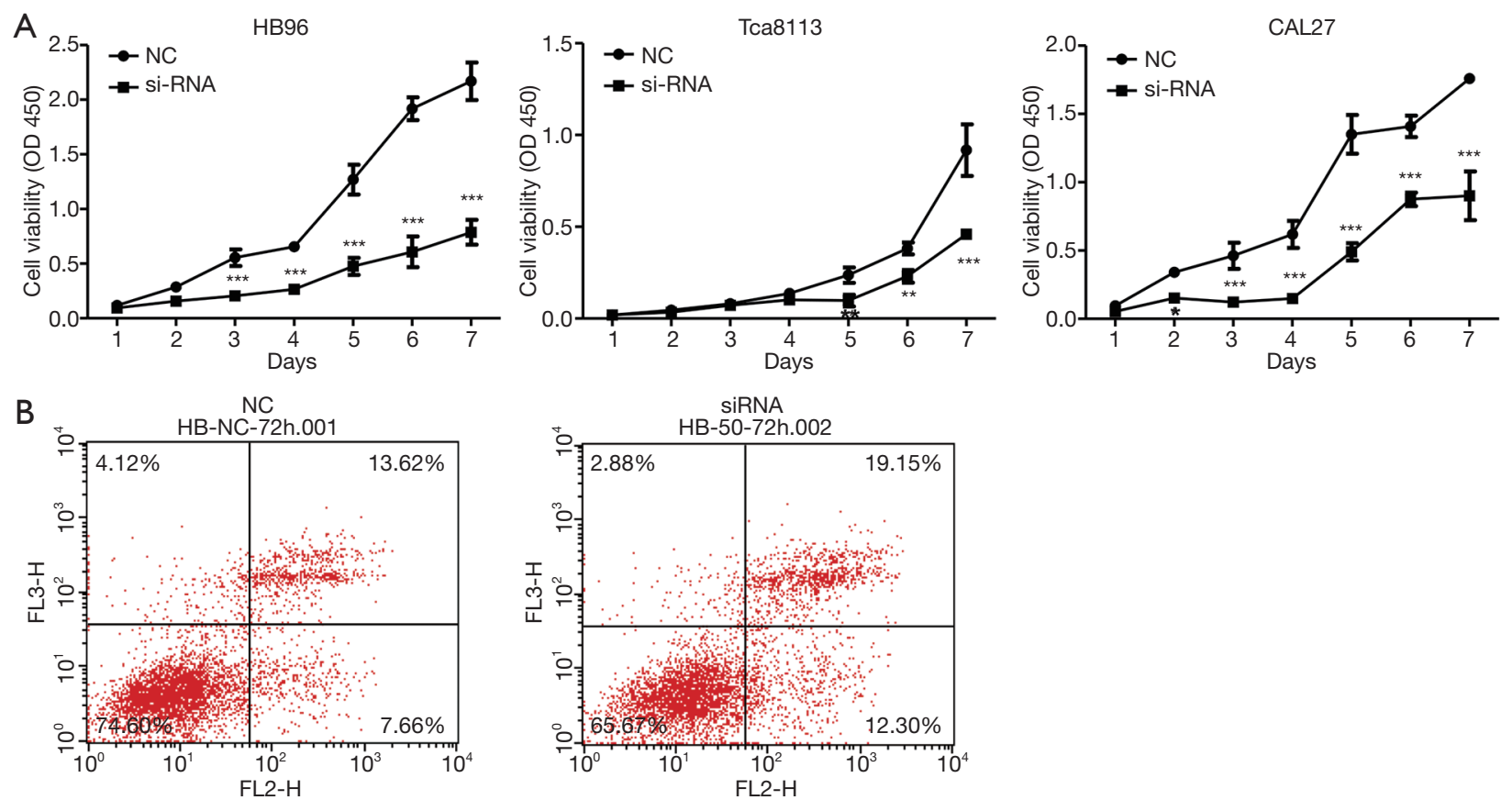

Figure 2 Effect of IGFBP3 knockdown on cell growth in HB96, CAL27, and Tca8113 cells. (A) Cell viability in HB96, CAL27, and Tca8113 cells transfected with si-IGFBP3-516 or siRNA-NC; (B) cell apoptosis in HB96 cells transfected with si-IGFBP3-516 or siRNANC. siRNA, small interfering RNA; IGFBP3, insulin-like growth factor binding protein 3 ; NC, negative control; **, $\mathrm{P}<0.01$; ***, $\mathrm{P}<0.001$.
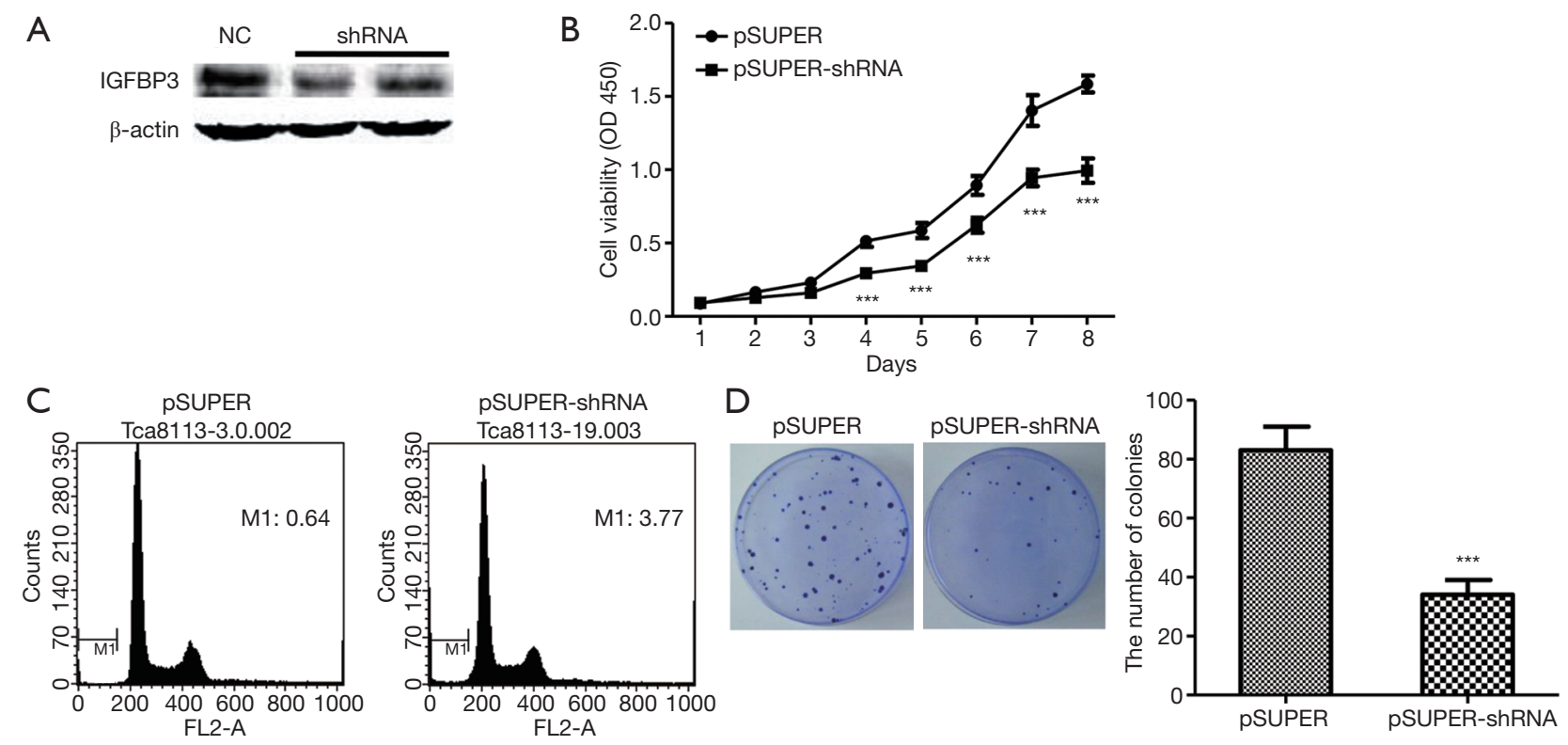

Figure 3 Effect of stable transfection of IGFBP3 knockdown on Tca8113 cell functions. (A) IGFBP3 protein expression in stably transfected Tca8113 cells; (B) cell viability in stably transfected Tca8113 cells; (C) cell apoptosis in stably transfected Tca8113 cells; (D) colony formation in stably transfected Tca8113 cells; shRNA, short hairpin RNA; IGFBP3, insulin-like growth factor binding protein 3 . ${ }^{* * *} \mathrm{P}<0.001$. 
A

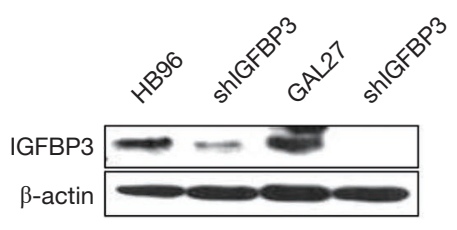

B

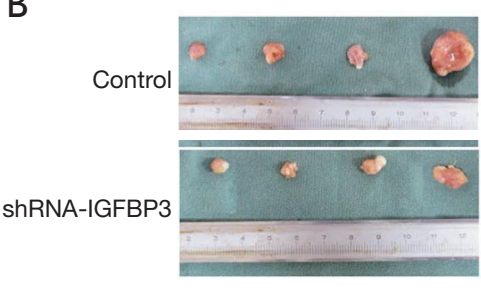

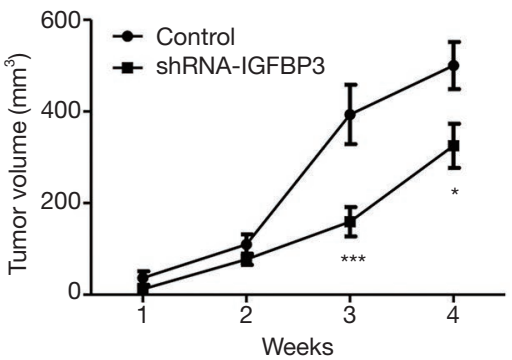

Figure 4 Effect of lentivirus-mediated overexpression of IGFBP3 on tumorigenesis. (A) IGFBP3 protein levels in HB96 and CAL27 cells infected with or without Lenti-shIGFBP3-EGFP; (B) tumor volume of xenografted IGFBP3-overexpressing CAL27 cells; IGFBP3, insulin-like growth factor binding protein 3; shRNA, short hairpin RNA. *, $\mathrm{P}<0.05$; *** $\mathrm{P}<0.001$.
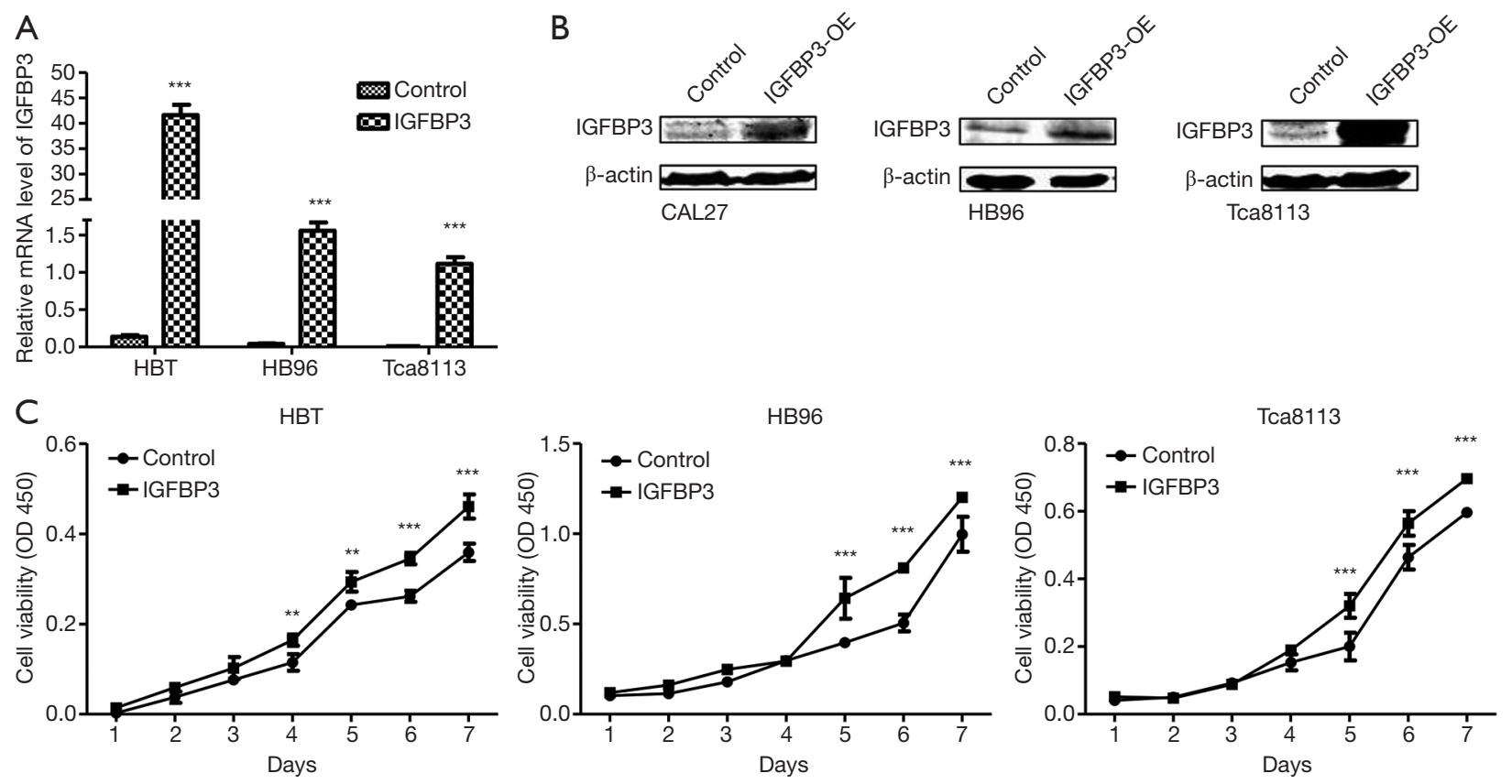

Figure 5 Effect of overexpressing IGFBP3 on cell viability. (A) mRNA expression of IGFBP3 in HB96, CAL27, and Tca8113 cells after transfection with or without pcDNA3.0-IGFBP3; (B) protein expression of IGFBP3 in HB96, CAL27, and Tca8113 cells after transfection with or without pcDNA3.0-IGFBP3; (C) cell viability of HB96, CAL27, and Tca8113 cells transfected with pcDNA3.0-IGFBP3 or empty vector; IGFBP3-OE, IGFBP3 overexpressing; IGFBP3, insulin-like growth factor binding protein $3 .{ }^{* *}, \mathrm{P}<0.05$; ${ }^{* *}, \mathrm{P}<0.001$.

\section{Effect of IGFBP3 overexpression on cell viability}

We constructed pcDNA3.0-IGFBP3 plasmid to overexpress IGFBP3 in OSCC cells. We transfected pcDNA3.0IGFBP3 plasmid into HB96, CAL27, and Tca8113 cells and found that IGFBP3 expression was significantly increased at both the mRNA and protein level in comparison with the control (Figure $5 A, B$ ). Taken together, the transfection efficiency of pcDNA3.0-IGFBP3 in HB96, CAL27, and
Tca8113 cells was significant to be used in our subsequent studies. Additionally, since silencing IGFBP3 inhibited cell growth, we further detected the effect of overexpressing IGFBP3 on cell viability. Figure $5 C$ shows that cell viability was increased in response to IGFBP3 overexpression in HB96, CAL27, and Tca8113 cells. These results demonstrated that overexpressing IGFBP3 promoted cell viability. 


\section{Discussion}

OSCC is one of the most common malignant tumors of the head and neck, whose morbidity is increasing in recent years. At present, surgical resection, radiotherapy and chemotherapy are the main treatments for OSCC, but the prognosis is still poor $(1,2)$. Therefore, the exploration of the molecular mechanism of OSCC to find new therapeutic targets and new diagnostic markers is of great significance. Along with the deepening of research, various biomarkers have been distinguished to be related with OSCC. For example, insulin-like growth factor-II mRNA binding protein-3 (IGF2BP3) was reported to be an independent predictor of death of patients with OSCC (19). Clauditz et al. also reported IGF2BP3 as an independent prognostic factor for squamous cell carcinomas of the head and neck, especially the subgroup of OSCC (20). Moreover, Hwang et al. showed that IGF2BP3 may be involved in the pathogenesis of OSCC, especially in the bone invasion (21). IGFBP3 is main component of the IGFBP family. Accumulating evidence suggest that IGFBP3 is the key factor in OSCC cell lines and OSCC models. Zhong et al. demonstrated the accumulation of IGFBP3 mRNA and protein in OSCC in vitro and in vivo, the expression of which correlated with the development of OSCC (12). In line with this study, IGFBP3 was further found to be sufficient to promote the migration, transmigration, and lymph node metastasis of OSCC cells, independent of the IGF/IGF1R axis (15). Therefore, IGFBP3 may act as a specific and positive biomarker for OSCC development and progression.

However, little is known about the direct and integrated role of IGFBP3 in OSCC oncogenesis at the molecular and cellular levels. The emergence and maturity of siRNA and gene cloning technology have provided effective approaches for exploring gene functions. In the present paper, we silenced IGFBP3 by siRNA-IGFBP3 and overexpressed IGFBP3 using the pcDNA3.0-IGFBP3 vector to dissect the effect of IGFBP3 in OSCC cells in vitro. Our results showed that transient silencing of IGFBP3 statistically impaired cell viability and enhanced cell apoptosis, while overexpressing IGFBP3 promoted cell viability in vitro. Although IGFBP3 is overexpressed in OSCC models, cell lines, and tissues, indicating that IGFBP3 may contribute to tumor cell growth and proliferation, OSCC cells intrinsically possess strong proliferative potential $(22,23)$. Furthermore, stable silencing of IGFBP3 diminished cell viability and colony formation, and elevated apoptosis in stably transfected cells. These findings indicated the role of IGFBP3 in promoting the cell growth of OSCC. These findings are consistent with a number of published reports, which have demonstrated IGFBP3 enhancement of cell proliferation by IGF-dependent and -independent mechanisms $(24,25)$. Thus, IGFBP3, acting as a tumor promoter, increased OSCC cell growth.

Given the fact that plasmid liposomes transfected into cells to change gene expression are prone to express gene loss and lower transfection efficiency $(26,27)$, we employed lentiviral transfection in vivo to confirm IGFBP3 stable expression without gene loss. CAL27-stable cell line with siRNA-IGFBP3 was subcutaneously injected into BALB/ cASlac-nu mice to observe tumor formation. Interestingly, no significant difference was observed in the size or weight of implanted tumors in the control or experimental group for 3 weeks. The difference between the transfection group and control group was significant from 3 to 4 weeks. This difference may be because of different tumor cell microenvironments in vitro and in vivo. In addition, the effect on tumor formation of interfering only one gene was limited. Hence, our study demonstrated that silencing IGFBP3 inhibited tumor formation. Intensive studies are encouraged to be performed to uncover the underlying mechanism of IGFBP3 function in OSCC tumorigenesis.

Collectively, silencing IGFBP3 significantly inhibited cell viability and colony formation, and promoted cell apoptosis in OSCC cells, as well as diminished tumorigenesis in nude mice. Taken together, our study described the protumorigenic role of IGFBP3 in OSCC and outlined a functional role for dysregulated IGFBP3 in OSCC cell growth. Therefore, IGFBP3 should be considered a novel gene target for OSCC treatment.

\section{Acknowledgments}

Funding: This study was supported by the National Natural Science Foundation of China (No. 81602370 $\&$ No.81802697) and The Interdisciplinary Program of Shanghai Jiao Tong University (No. YG2016QN16).

\section{Footnote}

Conflicts of Interest: All authors have completed the ICMJE uniform disclosure form (available at http://dx.doi. org/10.21037/tcr.2019.08.13). The authors have no conflicts of interest to declare. 
Ethical Statement: The authors are accountable for all aspects of the work in ensuring that questions related to the accuracy or integrity of any part of the work are appropriately investigated and resolved. This study was conducted in accordance with the Declaration of Helsinki (as revised in 2013). The institutional ethical approval and individual informed consent were waived.

Open Access Statement: This is an Open Access article distributed in accordance with the Creative Commons Attribution-NonCommercial-NoDerivs 4.0 International License (CC BY-NC-ND 4.0), which permits the noncommercial replication and distribution of the article with the strict proviso that no changes or edits are made and the original work is properly cited (including links to both the formal publication through the relevant DOI and the license). See: https://creativecommons.org/licenses/by-nc-nd/4.0/.

\section{References}

1. Bagan J, Sarrion G, Jimenez Y. Oral cancer: clinical features. Oral Oncol 2010;46:414-7.

2. van $\mathrm{Zyl} A$, Bunn BK. Clinical features of oral cancer. SADJ 2012;67:566-9.

3. Li L, Li C, Wang S, et al. Exosomes Derived from Hypoxic Oral Squamous Cell Carcinoma Cells Deliver miR-21 to Normoxic Cells to Elicit a Prometastatic Phenotype. Cancer Res 2016;76:1770-80.

4. Patel SG, Amit M, Yen TC, et al. Lymph node density in oral cavity cancer: results of the International Consortium for Outcomes Research. Br J Cancer 2013;109:2087-95.

5. Duan Y, Zhang S, Wang L, et al. Targeted silencing of CXCR4 inhibits epithelial-mesenchymal transition in oral squamous cell carcinoma. Oncol Lett 2016;12:2055-61.

6. Jogie-Brahim S, Feldman D, Oh Y. Unraveling insulin-like growth factor binding protein-3 actions in human disease. Endocr Rev 2009;30:417-37.

7. Schaeffer DF, Owen DR, Lim HJ, et al. Insulin-like growth factor 2 mRNA binding protein 3 (IGF2BP3) overexpression in pancreatic ductal adenocarcinoma correlates with poor survival. BMC cancer 2010;10:59.

8. Dupart JJ, Trent JC, Lee HY, et al. Insulin-like growth factor binding protein-3 has dual effects on gastrointestinal stromal tumor cell viability and sensitivity to the antitumor effects of imatinib mesylate in vitro. Mol Cancer 2009;8:99.

9. Lin MZ, Marzec KA, Martin JL, et al. The role of insulinlike growth factor binding protein-3 in the breast cancer cell response to DNA-damaging agents. Oncogene 2014;33:85-96.

10. Gigek CO, Leal MF, Lisboa LC, et al. Insulin-like growth factor binding protein-3 gene methylation and protein expression in gastric adenocarcinoma. Growth Horm IGF Res 2010;20:234-8.

11. Lin WH, Martin JL, Marsh DJ, et al. Involvement of insulin-like growth factor-binding protein-3 in the effects of histone deacetylase inhibitor MS-275 in hepatoma cells. J Biol Chem 2011;286:29540-7.

12. Zhong LP, Yang X, Zhang L, et al. Overexpression of insulin-like growth factor binding protein 3 in oral squamous cell carcinoma. Oncol Rep 2008;20:1441-7.

13. Natsuizaka M, Kinugasa H, Kagawa S, et al. IGFBP3 promotes esophageal cancer growth by suppressing oxidative stress in hypoxic tumor microenvironment. Am J Cancer Res 2014;4:29-41.

14. Natsuizaka M, Ohashi S, Wong GS, et al. Insulin-like growth factor-binding protein-3 promotes transforming growth factor-\{beta\}1-mediated epithelial-to-mesenchymal transition and motility in transformed human esophageal cells. Carcinogenesis 2010;31:1344-53.

15. Yen YC, Hsiao JR, Jiang SS, et al. Insulin-like growth factor-independent insulin-like growth factor binding protein 3 promotes cell migration and lymph node metastasis of oral squamous cell carcinoma cells by requirement of integrin beta1. Oncotarget 2015;6:41837-55.

16. Zhu DW, Yang X, Yang CZ, et al. Annexin A1 downregulation in oral squamous cell carcinoma correlates to pathological differentiation grade. Oral Oncol 2013;49:542-50.

17. Supriatno, Harada K, Hoque MO, et al. Overexpression of p27 Kip1 induces growth arrest and apoptosis in an oral cancer cell line. Oral Oncol 2002;38:730-6.

18. Udo H. An Alternative Method to Facilitate cDNA Cloning for Expression Studies in Mammalian Cells by Introducing Positive Blue White Selection in Vaccinia Topoisomerase I-Mediated Recombination Plos One 2015;10:e0139349.

19. Tarsitano A, Asioli S, Morandi L, et al. Laminin-5 and insulin-like growth factor-II mRNA binding protein-3 (IMP3) expression in preoperative biopsy specimens from oral cancer patients: Their role in neural spread risk and survival stratification. J Craniomaxillofac Surg 2016;44:1896-902.

20. Clauditz TS, Wang CJ, Gontarewicz A, et al. Expression of insulin-like growth factor II mRNA-binding protein 3 
in squamous cell carcinomas of the head and neck. J Oral Pathol Med 2013;42:125-32.

21. Hwang YS, Ahn SY, Moon S, et al. Insulin-like growth factor-II mRNA binding protein-3 and podoplanin expression are associated with bone invasion and prognosis in oral squamous cell carcinoma. Arch Oral Biol 2016;69:25-32.

22. Chen L, Ni S, Li M, et al. High Expression of BCCIP beta Can Promote Proliferation of Esophageal Squamous Cell Carcinoma. Dig Dis Sci 2017,62:387-95.

23. Cheng Y, Wang Y, Li J, et al. A novel read-through transcript JMJD7-PLA2G4B regulates head and neck squamous cell carcinoma cell proliferation and survival. Oncotarget 2017;8:1972-82.

24. Steiger-Luther NC, Darwiche H, Oh SH, et al. Insulin-

Cite this article as: $\mathrm{Xu} \mathrm{HY}$, Zhu DW, Zhong LP, Zhang ZY, Yang CZ, Yang X, Zhang P. Effects of insulin-like growth factor binding protein 3 on cell growth and tumorigenesis in oral squamous cell carcinoma. Transl Cancer Res 2019;8(5):1709-1717. doi: $10.21037 /$ tcr.2019.08.13 like growth factor binding protein-3 is required for the regulation of rat oval cell proliferation and differentiation in the 2AAF/PHX model. Hepat Med 2010;2010:13-32.

25. Ko JY, Yoo KH, Lee HW et al. Mxi1 regulates cell proliferation through insulin-like growth factor binding protein-3. Biochem Biophys Res Commun 2011;415:36-41.

26. Liu G, Wang Y, Hu Y, et al. Functionalized Multi-Wall Carbon Nanotubes Enhance Transfection and Expression Efficiency of Plasmid DNA in Fish Cells. Int J Mol Sci 2016;17:335.

27. Vernon MM, Dean DA, Dobson J. DNA Targeting Sequence Improves Magnetic Nanoparticle-Based Plasmid DNA Transfection Efficiency in Model Neurons. Int J Mol Sci 2015;16:19369-86. 